\title{
Birth Defect
}

National Human Genome Research Institute (NHGRI)

\section{Source}

National Human Genome Research Institute (NHGRI). Birth Defect.

A birth defect is an abnormality present at birth. Also called a congenital defect, it can be caused by a genetic mutation, an unfavorable environment during pregnancy, or a combination of both. The effect of a birth defect can be mild, severe, or incompatible with life. 